\title{
Ciclos de reforma y contra-reforma en la política de competencia en España*
}

\author{
Joan-Ramon Borrell Arqué \\ Universitat de Barcelona \\ Juan Luis Jiménez González \\ Universidad de Las Palmas de Gran Canaria \\ Carmen García Galindo \\ European University Institute
}

\section{Resumen}

Las políticas e instituciones están sometidas a ciclos de reforma y contra-reforma. Éstas pueden impulsar periodos de auge o declive en la efectividad de las políticas públicas. Estos ciclos son especialmente volátiles y amplios en los países que tienen problemas de estabilidad institucional. En España, la política de competencia ilustra estos ciclos volátiles y amplios en la efectividad de las políticas públicas como pocas otras. En los últimos 20 años (1995-2014), España ha vivido dos ciclos completos de intensos auges y declives en la efectividad de la política de competencia, con una ganancia media de efectividad del 14 por 100. El último ciclo de diez años (2005-2014) ha recogido un aumento destacable y posterior caída en la efectividad de la política de competencia, saldándose sin ganancia significativa en la misma: un inane y desgastante ciclo completo de reforma y contra-reforma. La comparación de resultados con un conjunto de 54 países del mundo muestra que esta volatilidad cíclica en la efectividad de la política de competencia en España es una anormalidad estadística. Dada la relevancia del marco institucional y el enforcement, nuestros resultados apuntan a la necesidad de estabilizar la calidad institucional en materia de competencia como garante de una mayor efectividad de la misma.

Palabras clave: $C N C, C N M C$, efectividad política de competencia, marco institucional.

Clasificación JEL: K21, K42, L41, L51.

\begin{abstract}
Policies and institutions are subject to reform and counter-reform cycles. These can stimulate periods of booms or decline in the effectiveness of public policies. These cycles are particularly volatile and large in countries that encounter institutional stability problems. In Spain, competition policy illustrates these volatile and large cycles in the effectiveness of public policies like few others. In the last 20 years (1995-2014), Spain has experienced two complete cycles of intense booms and declines in the effectiveness of competition policy, with an average of 14 por 100 effective gain. The last decade (2005-2014) has collected a remarkable rise and subsequent fall in the effectiveness of competition policy, settling without significant gain on it: an inane and

* Los autores quieren agradecer a Madeleine Hediger y a William Milner (IMD, World Competitiveness Center) por facilitarnos la información actualizada del indicador de efectividad de la competencia, así como a José Manuel Ordóñez (Universidad de Málaga) y a un evaluador anónimo por sus comentarios. No obstante, cualquier error u omisión es de exclusiva responsabilidad de los autores.
\end{abstract}


exhausting cycle of reforms and counter-reforms. The comparison of results with a set of 54 countries around the world shows that this cyclical volatility in the effectiveness of the competition policy in Spain is a statistical abnormality. Given the importance of the institutional framework and the enforcement, our results point to the need of stabilizing the institutional quality in the area of competition as a guarantor of greater effectiveness of the competition policy.

Keywords: CNC, CNMC, antitrust effectiveness, institutions.

JEL classification: K21, K42, L41, L51.

\section{Introducción}

Los beneficios de la competencia para el crecimiento económico o la productividad están ampliamente testados en la literatura económica (véase por ejemplo Dutz y Hayri; 2000; o Borrell y Tolosa, 2008). Es por ello que la efectividad de un sistema de política de la competencia es clave para garantizar los beneficios derivados de la misma: reducción de costes, selección natural del mercado, facilitador de la entrada de rivales e, incluso, mejora la eficiencia dinámica y difusión de nuevas tecnologías (Aghion y Howitt, 1996).

Dicha efectividad se ve afectada por diversos factores, algunos de ellos exógenos y otros propios del diseño y aplicación de las políticas de competencia (Borrell y Jiménez, 2008): el nivel de renta (en términos medios, mayor efectividad de la política de competencia cuanto más desarrollado es el país); pertenencia a la Unión Europea; introducción de programas innovadores en la persecución de los ilícitos restrictivos de la competencia, como el programa de clemencia (para una evaluación de dichos programas sobre la efectividad, véase Borrell, Jiménez y García, 2014); disposición de una autoridad de la competencia que garantice la independencia en la investigación y resolución de casos respecto de las partes interesadas y los poderes públicos (ejecutivo y legislativo).

Pero una pregunta previa debe ser: ¿cómo medir dicha efectividad? Desde hace unos años existe una literatura empírica que trata de acercarse a ese concepto a través de indicadores basados en encuestas de percepción de la efectividad de la política de competencia en un país. En general existen dos cuestionarios: el elaborado por el World Economic Forum (WEF, EEUU-China-Suiza) y el realizado por la Escuela de Negocios IMD (International Institute for Management Development, Suiza); y ambos plantean, entre otras preguntas, una de respuesta escalada con el tópico de competencia, en similar sentido: ¿considera que la política de competencia promueve de forma efectiva la competencia? Ambas variables están altamente correlacionadas (superan el 80 por 100) y ligadas igualmente a los niveles de renta de cada país (Voigt, 2009).

Este tipo de variables han sido utilizadas por la literatura académica como proxy de dicho nivel de competencia y de cómo las políticas sobre la materia han afectado al nivel de competencia percibido por los agentes económicos de cada país. Alternativamente, algunos autores han construido indicadores sintéticos cualitativos que aglutinan valoraciones de expertos de diversas características institucionales de 
la política de competencia en los países en un momento del tiempo que parecen estar asociadas a la efectividad, como por ejemplo Nicholson (2008) o Voigt (2009). Pero estos indicadores sintéticos no ofrecen series de datos tan largas y para un número de países tan elevado como las que ofrecen las encuestas de percepción de la efectividad, además de sufrir el problema habitual metodológico de cómo agregar los diversos indicadores cualitativos sobre las políticas que se analiza.

Como comentamos, son diversos los trabajos publicados que utilizan los datos de efectividad de la política de competencia a través de encuestas. Entre otros, el mencionado trabajo de Borrell y Jiménez (2008), los posteriores de Voigt (2009), Ma (2010), Ma (2012), Ma (2013) o el más reciente de Gutmann y Voigt (2014).

El objetivo del presente trabajo es mostrar la alta variabilidad de las medidas de efectividad percibida de la política de competencia en España, y cómo la amplitud de los ciclos de auge y declive de la política de competencia en España son una anormalidad estadística, ya que son más amplios a los que corresponderían al país por su nivel de desarrollo económico y social, y por la trayectoria de mejora de la política de competencia en las dos últimas décadas. La confirmación de esta hipótesis sustentaría un problema de mayor calado: la necesidad de una estabilidad e independencia del marco institucional como acicate a una correcta implementación de la política de competencia en España.

La estructura del trabajo es la siguiente. Tras esta breve introducción al análisis de la efectividad desde la perspectiva de los indicadores y la literatura que los aplica, la sección segunda describe los datos utilizados y la estrategia empírica seguida para tratar de alcanzar el objetivo propuesto. En la sección tercera mostraremos los resultados, en dos aproximaciones: descriptiva univariante y econométrica. Finalmente planteamos en la última sección las conclusiones e implicaciones para el debate sobre políticas públicas.

\section{Datos y método}

El World Competitiveness Center, que forma parte de la Escuela de Negocios IMD (International Institute for Management Development) con sede en Lausana (Suiza), lleva a cabo encuestas anuales (Business Opinion Survey) sobre la opinión de los ejecutivos de diferentes países sobre varios aspectos relacionados con la competitividad de los países.

La variable de efectividad que analizamos en este estudio proviene de dicha encuesta $I M D$, y es uno de los criterios por países que forman parte del World Competitiveness Yearbook. En concreto, analizamos el criterio 2.4.11: «Legislación de la Competencia» (Factor: Eficiencia del Gobierno; Sub-factor: Competencia y regulación) que pide a los encuestados una valoración del 1 al 6 sobre la siguiente afirmación en relación al país en el que han trabajado y vivido durante el último año: «La legislación de la competencia es efectiva a la hora de prevenir comportamientos anticompetitivos». El resultado promedio a nivel de país se traduce a una escala del 0 al 10 (siendo 10 el mayor valor de efectividad). 
El objetivo de las encuestas anuales es, entre otros, medir la percepción de la competitividad y el atractivo de cada país para inversores extranjeros. Las encuestas son enviadas en diferentes países a ejecutivos con altos cargos en distintas empresas. La muestra y su distribución son proporcionales a la aportación al Producto Interior Bruto de los distintos sectores económicos. Los ejecutivos deben evaluar preguntas relacionadas con la competencia que existe en el país en el que han vivido y han trabajado durante el último año. Esto permite obtener una visión en profundidad de la percepción presente y las expectativas que tienen dichos ejecutivos respecto al ambiente de competencia generado por las instituciones. Por ejemplo, en la encuesta realizada en el año 2014 (en el primer trimestre), IMD obtuvo 4.300 respuestas de 60 países diferentes.

$I M D$ facilita un panel no balanceado de datos relativos a la efectividad percibida por los ejecutivos de empresa encuestados de la competencia promedio correspondiente a 46 países en 1995, y durante 20 años hasta 2014. En este período, el panel ha ido incorporando países hasta que alcanzar los 60 en 2014.

El panel de datos no balanceado cuenta con 1.048 observaciones. En el Cuadro 1 se muestran los estadísticos descriptivos de la variable efectividad de la política competencia (IMD) junto a otras variables a nivel de la pareja país-año.

En este trabajo, nuestra estrategia empírica consiste en realizar un análisis de los ciclos a lo largo del tiempo de la efectividad en cada país respecto al resto de países de la muestra, para poder comparar tanto la amplitud como la frecuencia de los ciclos en España respecto a los países similares en términos de desarrollo económico y político. Esto es, realizamos una comparación con «iguales», lo que nos permita contextualizar la evolución de la competencia en nuestro país y, en una primera aproximación, plantear un punto de partida para valorar los últimos cambios institucionales acaecidos.

Asimismo, calculamos para cada país el cambio porcentual en el indicador de efectividad (Cambio IMD) para el conjunto de los 20 años, 1995-2014, y para cada uno de los dos períodos de 10 años en que se pueden dividir las series temporales,

CUADRO 1

ESTADÍSTICOS DESCRIPTIVOS

\begin{tabular}{|l|c|c|c|c|c|}
\hline \multicolumn{1}{|c|}{ Variable } & $\begin{array}{c}\text { Obser- } \\
\text { vaciones }\end{array}$ & Media & $\begin{array}{c}\text { Desviación } \\
\text { estándar }\end{array}$ & Mínimo & Máximo \\
\hline IMD & 1.048 & 5,53 & 1,26 & 1,81 & 8,59 \\
\hline PIB per cápita & 1.101 & 20.942 & 19.789 & 383 & 112.028 \\
\hline UE (pertenencia a) & 1.200 & 0,34 & - & 0 & 1 \\
\hline NAFTA (pertenencia a) & 1.200 & 0,05 & - & 0 & 1 \\
\hline OCDE (pertenencia a) & 1.200 & 0,57 & - & 0 & 1 \\
\hline
\end{tabular}

FUENTE: Elaboración propia. 
1995-2004 y 2005-2014. Y también calculamos el rango de la amplitud de los ciclos (Rango IMD) como la diferencia porcentual entre el valor máximo y mínimo del indicador de efectividad para cada país en cada período temporal.

La comparación gráfica de los ciclos y del rango respecto al cambio porcentual en la efectividad de la política de competencia (IMD) nos permite extraer conclusiones sobre las diferencias entre la serie temporal del indicador en España respecto al resto de países de la muestra (si las hubiera).

\section{Resultados}

En el Gráfico 1 podemos observar la evolución del indicador de efectividad de la política de competencia en el periodo 1995 a 2014, para España versus un conjunto de agrupaciones regionales de países para los que tenemos datos completos para todo el período ${ }^{1}$.

En el Gráfico 1 comparamos la serie de efectividad de la política de competencia en España respecto al grupo de 19 países de la Unión Europea para los que tenemos datos de 1995 a 2014 (excluimos en el análisis gráfico los datos de los 7 países de la UE para los que tenemos datos para algunos años, mientras que no disponemos de datos de 2 países adicionales de los 28 que actualmente forman parte de la UE).

También comparamos la serie de efectividad de España respecto a los 30 países de la OCDE para los que tenemos series completas de 1995 a 2014, de los 34 países que actualmente forman parte de la OCDE.

El primer resultado destacado es el comportamiento tendencial similar para los valores medios agregados para la UE-19 y la OCDE-30, de crecimiento entre 1995 y 2002-2003, caída suave hasta 2010 , crecimiento posterior hasta 2012 y decrecimiento en 2013-2014. Se observan así ciclos de mejora y declive en la efectividad de la política de competencia para todos ellos.

Un hecho destacado es que en el caso de España, la variable de efectividad tiene unos ciclos mucho más pronunciados que el resto, tanto en las caídas como en las mejoras.

La percepción de la efectividad tiene un pronunciado auge de la percepción de efectividad del período 2006-2011 que coincide con la discusión y aprobación de la nueva Ley de Competencia de 2007 y la creación de la Comisión Nacional de Competencia; así como el abrupto decrecimiento después de 2011.

Aunque las encuestas del $I M D$ se envían en enero a los ejecutivos y deben completarse antes del mes de abril de cada año, el dato de 2012 recoge ya el cambio institucional que se produjo en 2013. En marzo de 2012 la Comisión Nacional de

\footnotetext{
${ }^{1}$ No incorporamos en estas agrupaciones a países para los que sólo tenemos datos para los últimos años del panel, y de esta manera evitamos que la serie esté afectada por los cambios en la muestra de países que representa.
} 


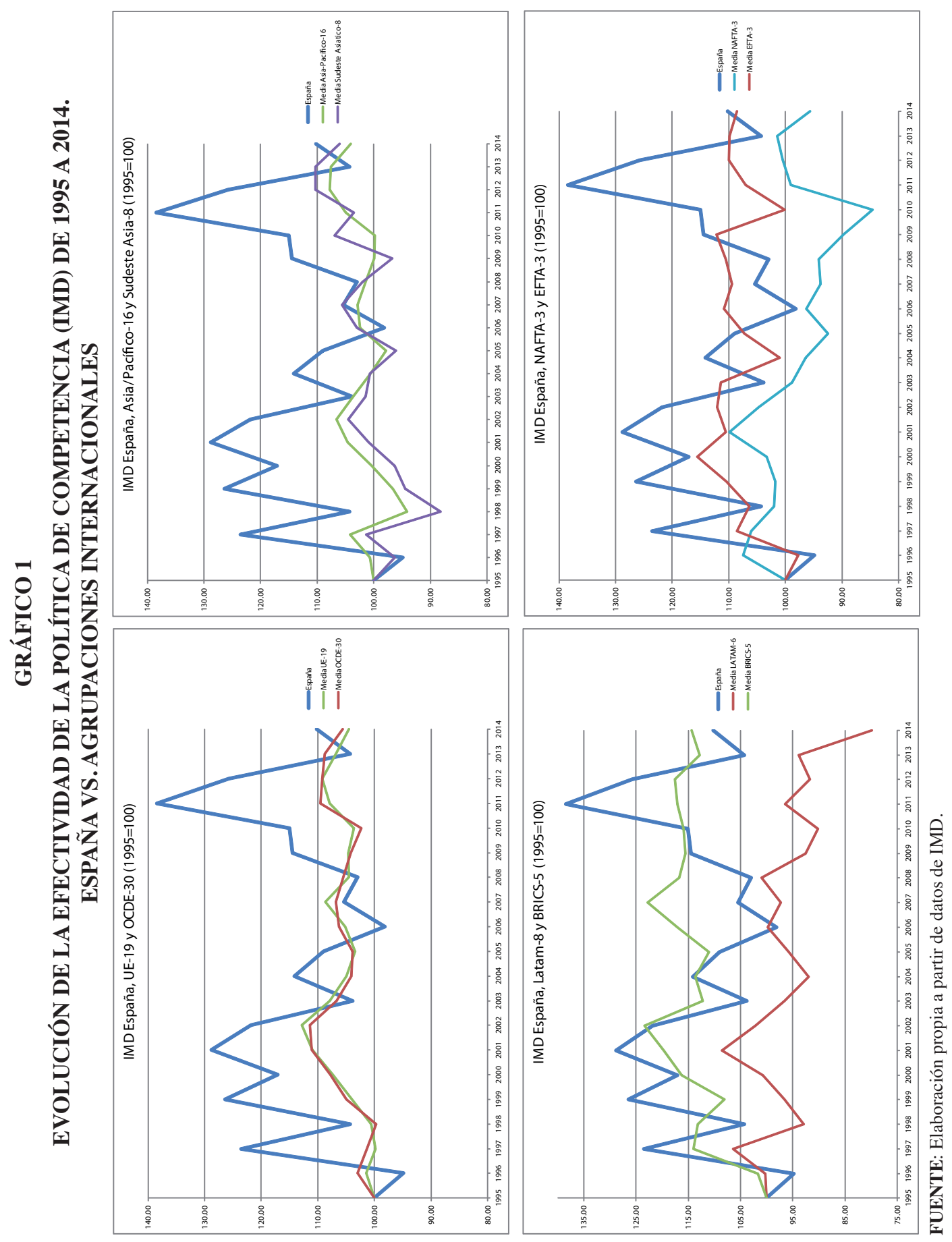


la Competencia emitió un informe sobre el anteproyecto de Ley de creación de la nueva Comisión Nacional de los Mercados y la Competencia, que no se puso en marcha hasta 2013. Así, las encuestas parecen recoger de forma negativa el cambio institucional en la Autoridad de Competencia del período 2012-2013, con un leve rebote en 2014.

El promedio de mejora del indicador de efectividad es de un 10 por 100 aproximadamente, tanto en el caso de España como en la UE-19 y la OCDE-30. Sin embargo, el análisis del Gráfico 1 muestra las diferencias en la amplitud de los ciclos.

Esta diferencia entre la gran amplitud de los ciclos en la percepción de efectividad en España respecto al ciclo promedio en los grupos UE-19 y la OCDE-30, también se observa en el gráfico que compara la serie española con las series promedio de la agrupación Asia/Pacífico-16 (16 países), y la agrupación Sudeste Asiático-8 (8 países).

De nuevo, la mejora promedio es aproximadamente del 10 por 100 tanto en España, como en las agrupaciones de Asia/Pacífico-16 y del Sudeste Asiático-8, pero de nuevo destaca la amplitud de los ciclos de la efectividad de la política de competencia en España en relación a las series de estas agrupaciones.

Las diferencias de amplitud en los ciclos de la percepción de efectividad de la política de competencia no parecen significativas en el caso de la comparación entre España y el grupo de 6 países de América Latina (LATAM-6: Argentina, Brasil, Chile, Colombia, México y Venezuela), ni con el grupo de 5 países emergentes (BRICS-5: Brasil, Rusia, India, China y Sudáfrica).

Todo ello a pesar de las diferencias notables en cuanto a la experiencia en materia de política de competencia de España frente a estos once países de referencia. Recordemos que la primera legislación de competencia en España es de 1964, aunque su diseño y aplicación fue completamente inefectiva, ya que obedeció a una lógica ajena a la promoción de la competencia en los mercados (véase Borrell, 1998). El verdadero inicio de la política de competencia en España hay que situarlo en 1989, bajo el impulso del proceso de armonización de la legislación española al acervo comunitario (acquis communautaire) acordado en el Tratado y Acta de Adhesión de España a la Unión Europea (entonces Comunidades Europeas).

Respecto a estas agrupaciones, hay además diferencias en el cambio de la efectividad a lo largo del tiempo. En LATAM-6, la percepción de efectividad cae un 20 por 100 aproximadamente entre 1995 y 2014, mientras que en España mejora un 10 por 100. En BRICS-5, la percepción de la efectividad mejora un 15 por 100, frente al 10 por 100 en España.

Las diferencias en la amplitud de los ciclos de la percepción de la efectividad de la política de competencia tampoco es tan importante respecto a los tres países del EFTA (Islandia, Noruega y Suiza), ni respecto a los tres países del NAFTA (Canadá, Estados Unidos y México). Tanto en España como en el grupo EFTA-3, la percepción de efectividad tiene una mejora similar del 10 por 100 para el período 1995 y 2014, mientras que la amplitud de los ciclos es ligeramente mayor en España que en EFTA- 
3. En los países del NAFTA-3, dicha percepción no mejora a lo largo del período 1995-2014, y de nuevo la amplitud de los ciclos es en España ligeramente mayor que en NAFTA-3.

El análisis de la diferencia en la amplitud de los ciclos entre España y las agrupaciones de países referidas anteriormente es, con todo, incompleto. Al agregar y obtener los promedios simples no ponderados de las series de efectividad de la política de competencia en varios países, obtenemos unas nuevas series que por definición estadística tendrán una amplitud menor, ya que los extremos de las series de los distintos países se reducirán al promediarse con los valores máximos y mínimos del resto de países.

Así pues, es necesario completar este análisis con la comparación de la serie de efectividad de la política de competencia en España con la de otros países de forma individualizada, para comprobar de esta manera si la amplitud de los ciclos es realmente mayor en España que en el resto de países de la muestra cuando emparejamos las series originales a nivel de cada país.

En esta comparación, es importante emparejar datos entre países similares, así como realizar algún análisis más detallado que nos permita comparar las variaciones de las series teniendo en cuenta algunas características de estas mismas series como, por ejemplo, la trayectoria de mejora o deterioro de la serie de efectividad durante el período observado para cada país.

El Gráfico 2 muestra la comparación de las series con cuatro países para los que la percepción de efectividad de la política de competencia entre 1995 y 2014 mejora aproximadamente en un 10 por 100 tal y como lo hace en España: Francia, Reino Unido, Holanda y Suecia.

A pesar de que estos cuatro países incluidos en el Gráfico 2 también sufren variaciones importantes de la percepción de efectividad de la política de competencia a lo largo de estas dos décadas, las diferencias en la amplitud de los ciclos para recorrer una misma trayectoria de mejora de la efectividad de la política de competencia son bien visibles.

Adicionalmente, para aprovechar la riqueza de los datos de los que disponemos, comparamos la relación entre la variabilidad en las series de percepción de efectividad de la política de competencia a nivel de país y el cambio (mejora o declive) promedio para el conjunto del período 1995 a 2014, además de diferencias para la primera (1994-2004) y segunda década (2005-2014) considerada.

En estas comparaciones incorporamos los datos relativos a todos los países para los que disponemos datos para el conjunto del período (46 países), para la primera década (también 46 países), y para la segunda década (54 países).

En este análisis restringido a 46 o 54 países, tomamos los datos de los países para los que tenemos la serie temporal completa (1995-2014), y también las series de Islandia (para el que falta sólo el dato de un año de la segunda década (2004-2014) y Qatar (sólo en la segunda década, 2004-2014, a pesar de que faltan 3 de los 10 datos de este período analizado). 

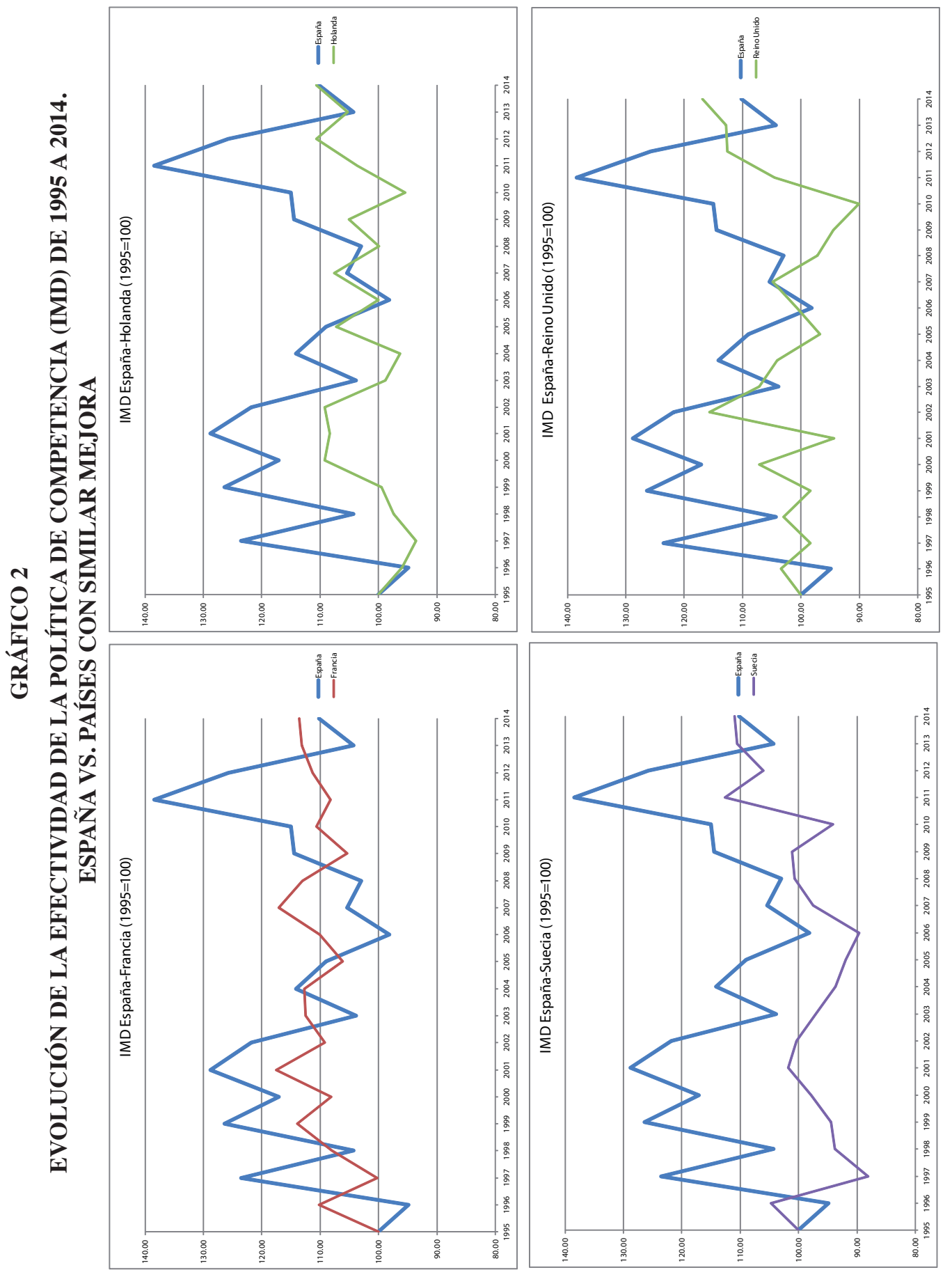

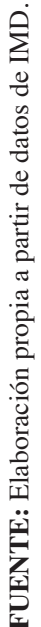


En los Gráficos 3 a 5 comparamos la amplitud del rango entre el valor máximo y mínimo de cada serie en porcentaje respecto a la magnitud de la mejora o declive del indicador de efectividad desde el inicio hasta el final de cada período analizado también en porcentaje. Los gráficos muestran tanto la dispersión de los datos como la mejor predicción y el rango de predicción del 95 por 100 de una regresión no paramétrica polinómica fraccionada del tipo:

$$
y_{i}=\beta_{0}+\beta_{1} x_{i}^{(p 1)}+\beta_{2} x_{i}^{(p 2)}+u_{i}
$$

donde se escogen las potencias ( 1 y p2) que mejor se ajustan a los datos (combinaciones de dos en dos de potencias como $-2,-1,-0.5,0,0.5,1,2,3$; donde 0 indica transformación logarítmica). En el polinomio, $y$ es el rango de variación en el indicador de efectividad, y $x$ es el cambio (mejora o declive) en el indicador de efectividad.

A priori esperamos que el rango de variación de los datos de la serie de cada país sea una función creciente respecto al cambio en valor absoluto en la serie del indicador de efectividad de la política de competencia en cada país. Ahora bien, la predicción del polinomio, así como el intervalo de confianza de dicha predicción al 95 por 100, nos muestra qué países tiene un rango de variación en los datos fuera de la normalidad estadística.

Tal y como muestra el Gráfico 3, los datos apuntan esta relación creciente entre el rango de variación de las series y el cambio de inicio a final del período 1995 a 2014. Para el conjunto del período, la serie correspondiente a España queda un poco por encima del intervalo de confianza de la predicción al 95 por 100.

Este resultado muestra que España tiene un anormalmente elevado rango de variación en la serie de efectividad de la política de competencia. Esta anormalidad estadística la comparte con países como Islandia, China, Hong Kong, Hungría, Colombia, República Checa, México, Indonesia, Argentina y Polonia. Para los países del entorno de España, de la UE o incluso parte de la propia OCDE, los resultados muestran cómo éstos se sitúan bien dentro del intervalo de confianza, o incluso por debajo de éste (véase Francia, Suecia o Reino Unido de forma destacada en el Gráfico 3). 


\section{GRÁFICO 3}

IMD: RANGO DE VARIACIÓN Y CAMBIO

(Período completo 1995-2014))

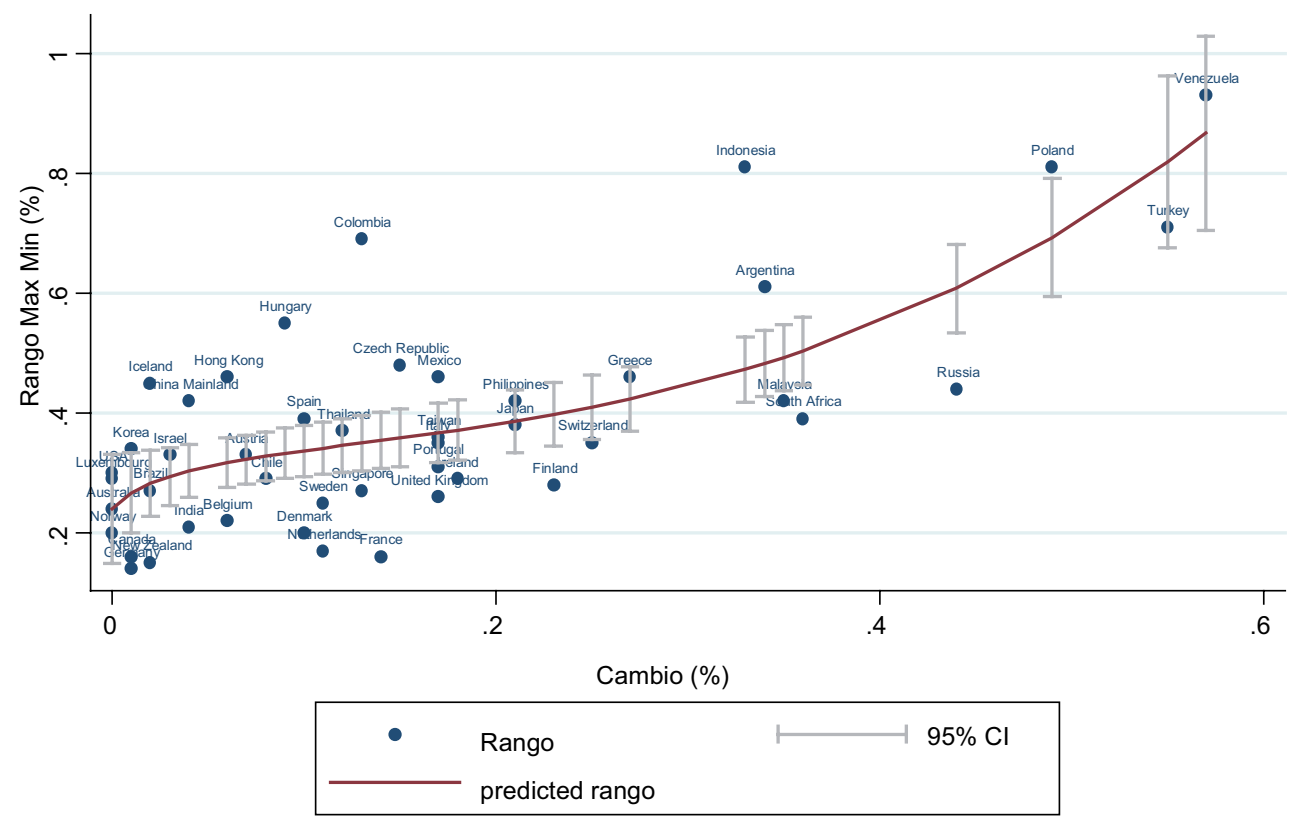

FUENTE: Elaboración propia. 
Por su parte, el Gráfico 4 muestra que en el período 1995-2004, el rango de variación para España en la serie de efectividad de la política de competencia estuvo dentro de la normalidad dado el cambio (mejora en este caso) en el indicador de efectividad.

\section{GRÁFICO 4 \\ IMD: RANGO DE VARIACIÓN Y CAMBIO \\ (Subperíodo 1995-2004)}

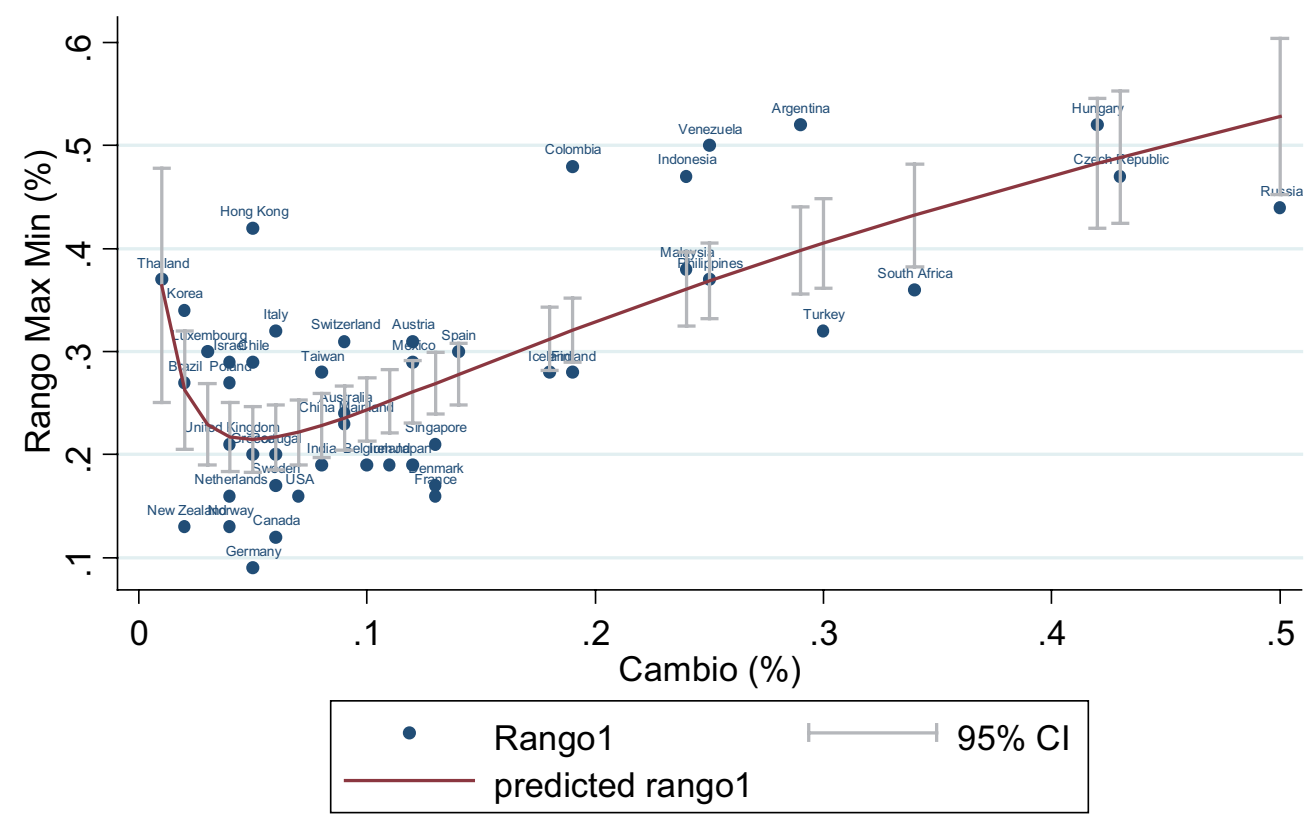

FUENTE: Elaboración propia. 
En cambio, el Gráfico 5 muestra que los datos de España quedan fuera de la normalidad en el período 2005 a 2014. En esta última década, la serie de efectividad de la política de competencia en España muestra un incremento promedio casi nulo (1 por 100), mientras que el rango entre los valores máximos y mínimos es muy elevado (36 por 100).

Comparte esa anormalidad de elevada amplitud en el ciclo de la política de competencia con otros países con escasos avances en la efectividad de la política de competencia como México, Jordania, Corea, Eslovenia, Filipinas y China. Y comparte esa amplitud en el ciclo también con Argentina, Austria, Portugal, Israel, Turquía, Islandia, Eslovaquia, Grecia, Bulgaria y Colombia.

\section{GRÁFICO 5}

\section{IMD: RANGO DE VARIACIÓN Y CAMBIO}

(Subperíodo 2005-2014)

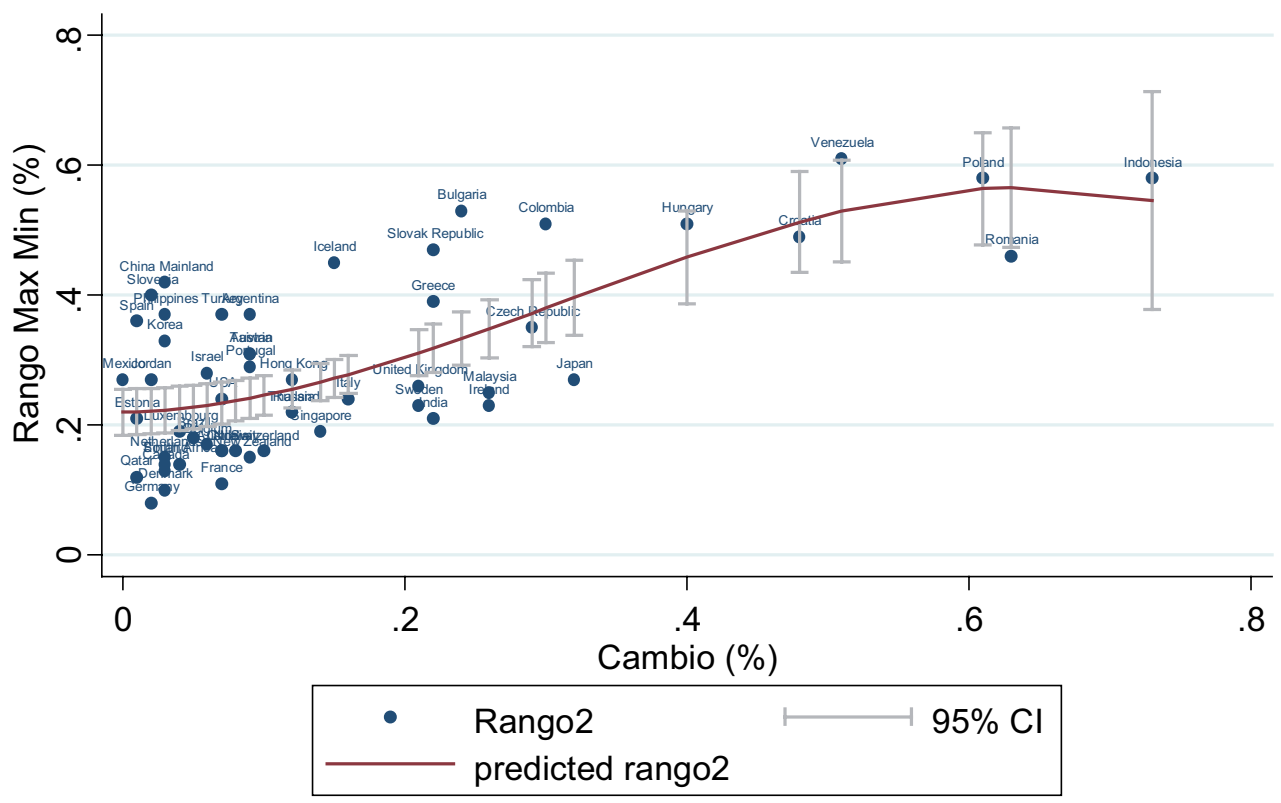

FUENTE: Elaboración propia. 
Finalmente, esa anormalidad estadística de España también se da para todo el período 1995-2014 si restringimos la muestra de países a los más ricos, los que tienen una renta per cápita en 2013 superior a los US\$20.000. En el Gráfico 6 la amplitud del ciclo de la efectividad de la política de competencia español supera el rango normal con gran diferencia, como sólo se alcanza en los casos de Islandia y Hong Kong.

\section{GRÁFICO 6}

IMD: RANGO DE VARIACIÓN Y CAMBIO. PAÍSES CON RENTA PER CÁPITA $>20.000$ \$

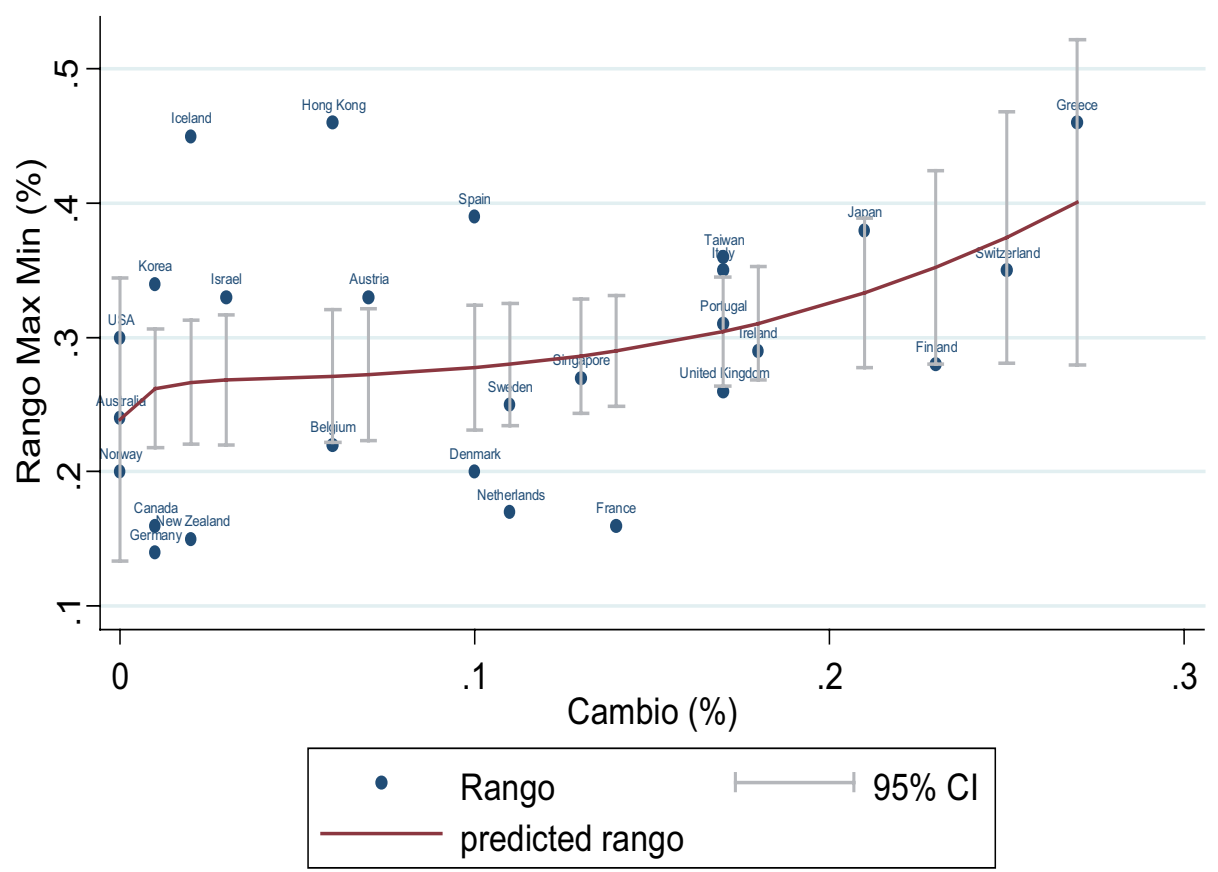

FUENTE: Elaboración propia.

Queda por determinar la influencia que puedan tener los procesos de fusión de organismos reguladores y de defensa de la competencia como el que se ha vivido en España o en México durante la última década en la que se producen estos resultados de anormal amplitud en el ciclo de la percepción de efectividad de la política de competencia, y escasa mejora en dicha efectividad.

Las similitudes y diferencias de los procesos de fusión de autoridades recientes han sido analizadas por Delgado y Mariscal (2014). Y otras experiencias de fusión de organismos de defensa de la competencia y de defensa del consumidor, así como 
de regulación como el de Holanda, no parecen haber tenido de momento el mismo impacto en la percepción de efectividad de la política de competencia que en España o en México.

\section{Conclusiones y discusión}

Este trabajo ha mostrado cómo la percepción de la efectividad de la política de competencia está sometido a intensos ciclos de mejora y declive, utilizando para ello una muestra de 54 países del mundo durante los últimos 20 años (1995-2014). Estos ciclos son más volátiles y amplios en algunos países de menor renta per cápita, y por ello, menor estabilidad económica, política e institucional. En estos países, las políticas e instituciones están sometidas a ciclos de reforma y contra-reforma que no siempre aportan mejoras significativas en la efectividad de las políticas públicas.

En España, la política de competencia ilustra estos ciclos más volátiles y amplios en la efectividad de las políticas públicas como pocas otras. En los últimos 20 años (1995-2014), España ha vivido dos ciclos completos de intensos auges y declives en la efectividad de la política de competencia con una ganancia de efectividad del 14 por 100 .

El último ciclo de diez años (2005-2014) de auge y caída en la efectividad de la política de competencia se ha saldado sin ganancia significativa en la efectividad: un inane y desgastante ciclo completo de reforma y contra-reforma.

Los datos de efectividad comparada con un conjunto de 54 países del mundo, muestra que esta volatilidad cíclica en la efectividad de la política de competencia es una anormalidad estadística, no se corresponde a la estabilidad institucional que muestran países con un nivel económico y social similar al que ha alcanzado España, ni a la amplitud de los ciclos que corresponde a países que han alcanzado cambios de la misma magnitud que los obtenidos por España en las dos últimas décadas.

A partir de esta evidencia, nuevos trabajos deberían dirigir nuevos esfuerzos a identificar bajo qué condiciones la interacción estratégica entre los agentes políticos y económicos conduce a algunos países hacia el bloqueo en equilibrios de bajo nivel de inútil reforma y contra-reforma (como muestran Aghion y Schankerman, 2004), mientras que otros países son capaces de transitar hacia equilibrios de alto nivel en los que las reformas permiten avanzar hacia mejoras significativas en la efectividad de la política de competencia.

\section{Referencias bibliográficas}

[1] AGHION, D. y HOWITT, P. (1996): «A model growth through creative destruction», Econometrica, 60, pp. 323-351.

[2] AGHION, P. y SCHANKERMAN, M. (2004): «A Model of the Private and Social Returns to Market-Enhancing Infrastructure», Economic Journal. 
[3] BORRELL, J. R. (1998): «Spanish competition policy: a case of government's response to domestically perceived problems», The Antitrust Bulletin, 43(2), pp. 445-465.

[4] BORRELL, J. R. y JIMÉNEZ, J. L. (2008): «The drivers of antitrust effectiveness». Hacienda Pública Española - Revista de Economía Pública, 185(2), pp. 69-88.

[5] BORRELL, J.R., JIMÉNEZ, J.L. y GARCÍA, C. (2014): «Evaluating antitrust leniency programmes», Journal of Competition Law \& Economics. 10(1), pp. 107-136.

[6] BORRELL, J. R. y TOLOSA, M. (2008): «Endogenous antitrust: cross country evidence on the impact of competition - enhancing policies on productivity», Applied Economic Letters, 15 (11), pp. 827-831.

[7] DELGADO, J. y MARISCAL, E. (2014): «Integrating regulatory and antitrust powers: does it work? Case studies from Spain and Mexico», Competition Policy International, 10(1), Spring, pp. 135-159.

[8] DUTZ, M. A., y HAYRI, A. (2000): «Does more intense competition lead to higher growth?» World Bank Policy Research Working Paper, 2320 and CEPR Discussion Paper No. 2249.

[9] GUTMANN, J. y VOIGT, S. (2014): «Lending a hand to the invisible hand? Assessing the effects of newly enacted competition laws». Available at SSRN: ssrn.com/ abstract $=2392780$

[10] MA, T.-C. (2010): «Competition authority independence, antitrust effectiveness, and institutions». International Review of Law Economics, 30, pp. 226-235.

[11] MA, T.-C. (2012): «Legal tradition and antitrust effectiveness». Empirical Economics, 43 (3), pp. 1263-1297.

[12] MA, T.-C. (2013): «Legal transplant, legal origin, and antitrust effectiveness». Journal of Competition Law \& Economics, 9(1), pp. 65-88.

[13] NICHOLSON, M. W. (2008): «An antitrust law index for empirical analysis of international competition policy». Journal of Competition Law \& Economics, 4(4), pp. 1009-1029.

[14] VOIGT, S. (2009): «The effect of competition policy on development: Cross-country evidence using four new indicators». Journal of Development Studies, 45(8), pp. 1225 1248. 